\title{
Comprehensive microRNA profiling of prostate cancer cells after ionizing radiation treatment
}

\author{
CHUNG-MAN LEUNG ${ }^{1,2}$, SUNG-CHOU LI ${ }^{3}$, TING-WEN CHEN ${ }^{4,5}$, MENG-RU HO $^{6}$, LING-YUEH HU ${ }^{7}$, \\ WEN-SHAN LIU ${ }^{1}$, TONY T. WU ${ }^{8,9}$, PING-CHI HSU ${ }^{2}$, HONG-TAI CHANG ${ }^{8,10}$ and KUO-WANG TSAI ${ }^{11}$ \\ ${ }^{1}$ Department of Radiation Oncology, Kaohsiung Veterans General Hospital, Kaohsiung; ${ }^{2}$ Department of Safety, \\ Health and Environmental Engineering, National Kaohsiung First University of Science and Technology, \\ Kaohsiung; ${ }^{3}$ Genomics and Proteomics Core Laboratory, Department of Medical Research, Kaohsiung Chang Gung \\ Memorial Hospital and Chang Gung University College of Medicine, Kaohsiung; ${ }^{4}$ Molecular Medicine Research Center \\ and ${ }^{5}$ Bioinformatics Center, Chang Gung University, Taoyuan; ${ }^{6}$ Genomics Research Center and ${ }^{7}$ Institute of Biomedical \\ Sciences, Academia Sinica, Taipei; ${ }^{8}$ Department of Surgery, Kaohsiung Veterans General Hospital, Kaohsiung; \\ ${ }^{9}$ School of Medicine, Yang-Ming University, Taipei; Departments of ${ }^{10}$ Emergency and ${ }^{11}$ Medical Education \\ and Research, Kaohsiung Veterans General Hospital, Kaohsiung, Taiwan, R.O.C.
}

Received November 21, 2013; Accepted December 23, 2013

DOI: $10.3892 /$ or.2014.2988

\begin{abstract}
MicroRNAs (miRNAs) are small, non-coding RNAs that negatively regulate gene expression and have emerged as potential biomarkers in radiation response to human cancer. Only a few miRNAs have been identified in radiation response to prostate cancer and the involvement of the radiation-associated miRNA machinery in the response of prostate cancer cells to radiation is not thoroughly understood. Therefore, the purpose of the present study was to comprehensively investigate the expression levels, arm selection preference and isomiRs of radiation-response miRNAs in radiation-treated $\mathrm{PC} 3$ cells using a next-generation sequencing (NGS) approach. Our data revealed that the arm selection preference and $3^{\prime}$ modification of miRNAs may be altered in prostate cancer after radiation exposure. In addition, the proportion of AA dinucleotide modifications at the end of the read gradually increased in a time-dependent manner after PC3 radiation treatment. We also identified 6 miRNAs whose expression increased and 16 miRNAs whose expression decreased after exposure to $10 \mathrm{~Gy}$ of radiation. A pathway enrichment analysis revealed that the target genes of these radiation-induced miRNAs significantly co-modulated the radiation response pathway, including the mitogen-activated protein kinase (MAPK), Wnt, transforming growth factor- $\beta$
\end{abstract}

Correspondence to: Dr Kuo-Wang Tsai, Department of Medical Education and Research, Kaohsiung Veterans General Hospital, Kaohsiung 813, Taiwan, R.O.C.

E-mail:kwtsai@vghks.gov.tw; kwtsai6733@gmail.com

Dr Hong-Tai Chang, Department of Surgery, Kaohsiung Veterans General Hospital, Kaohsiung 813, Taiwan, R.O.C.

E-mail: htchang@vghks.gov.tw

Key words: microRNA, prostate cancer, next-generation sequencing, pathway enrichment analysis, radiation, The Cancer Genome Atlas
(TGF- $\beta$ ) and ErbB signaling pathways. Furthermore, analysis of The Cancer Genome Atlas (TCGA) database revealed that the expression of these radiation-induced miRNAs was frequently dysregulated in prostate cancer. Our study identified radiation-induced miRNA candidates which may contribute to radiosensitivity and can be used as biomarkers for radiotherapy.

\section{Introduction}

Prostate carcinoma is the most frequently diagnosed visceral cancer in men worldwide. An increasing prevalence has been reported in recent decades (1). Radiation therapy is one of the primary modalities in prostate cancer treatment. Ionizing radiation damages cells through free radicals from the radiolysis of water that cause DNA double-strand breaks. However, the efficacy of the radiotherapy may be affected by the cellular response to radiation. Radiotherapy is highly effective in treating radiosensitive tumors and enhancing the therapeutic efficacy can increase the overall survival rate. However, the presence of radioresistant tumors leads to cancer relapse and metastasis. Understanding the tumor-radiation-related genes to predict the tumor response to radiotherapy may potentially modulate the treatment outcome for prostate cancer patients.

MicroRNAs (miRNAs) are a family of small, non-coding, single-stranded RNAs composed of $\sim 22$ nucleotides (nt) that negatively regulate protein expression at the post-transcriptional level (2). They function as gene regulators by binding to partially complementary sites of mRNAs and cause translation inhibition or direct degradation of the target mRNA. It has been suggested that miRNAs are responsible for controlling $\sim 50 \%$ of all protein-coding genes (3). The widespread regulation of protein levels has been studied in cellular models (4). Previous studies have demonstrated that the expression of miRNAs is clearly involved in cancer development, and the deregulation of several miRNAs has been observed in various 
types of cancer, including prostate cancer. Porkka et al (5) was the first to identify a miRNA signature specific for prostate cancer by systematically profiling prostate cancer cell lines. Numerous studies have identified many dysfunctional miRNAs by using a high-throughput approach, which contributed to prostate cancer progression, including the let-7 family, miR-1, $-20 \mathrm{a},-21,-34 \mathrm{a},-106 \mathrm{~b},-125 \mathrm{~b},-205$ and -521 (6-13). Although several studies have investigated the role of these dysfunctional miRNAs to develop prostate cancer therapy, few studies have determined the roles of miRNAs in radiation response in prostate cancer. The upregulation of miR-521 reduces the response to radiation damage by specifically targeting a DNA repair protein, the Cockayne syndrome protein A (13). Li et al (14) found that miR-106b was dysregulated after radiation treatment and suppressed radiation-induced p21 activation, suggesting it may override radiation-induced cell cycle arrest and cell growth inhibition. Radiation delivered in daily fractions altered a greater number of miRNAs compared with single-dose radiation, and involved the upregulation of miR-34a and let-7 miRNAs (15).

Next-generation sequencing (NGS) is a high-throughput screening technology, and NGS data can be applied in investigating miRNA expression, miRNA isoforms (isomiRs) and the arm selection preferences of miRNAs. Therefore, the purpose of the present study was to comprehensively investigate the distribution of miRNAs after radiation treatment in PC3 cells by using an NGS approach. Furthermore, we explored the function of radiation-associated miRNA by conducting an in silico analysis.

\section{Materials and methods}

Cell culture and radiation treatment. A PC3 cell line was obtained from the American Type Culture Collection and was maintained in RPMI-1640 and supplemented with $10 \%$ inactivated fetal bovine serum (FBS; Invitrogen, Carlsbad, CA, USA). The cells were exposed to various radiation dosages ( 0 , 2, 6, 10, 14 and $18 \mathrm{~Gy}$ ) and were subsequently cultured in fresh medium. The total RNA was obtained at various time points $(0,5,15$ and $40 \mathrm{~h}$ after treatment) by using TRIzol (Invitrogen) according to the manufacturer's instructions. The concentration, purity and amount of total RNA were determined using a NanoDrop 1000 spectrophotometer (NanoDrop Technologies, Inc., USA).

Collection and preprocessing of sequence reads. PC3 cells were exposed to $10 \mathrm{~Gy}$ of radiation. After radiation treatment, the cells were lysed at various time points $(0,5,15$ and $40 \mathrm{~h})$ for RNA extraction. The RNA samples were prepared using an Illumina small RNA preparation kit, and were subsequently sequenced using the Illumina HiSeq platform. The generated sequence reads were first subjected to quality control to remove low-quality reads. The sequence reads were then subjected to $3^{\prime}$ adaptor trimming to generate clean reads, as previously described $(15,16)$. To attain a high confidence level, only the clean reads with a read count $\geq 2$ and with a length ranging from 15 to $27 \mathrm{nt}$ were included in further analyses.

Mapping clean reads to pre-miRNAs. To investigate miRNA expression profiles in different libraries, we mapped the qualified clean reads back to human pre-miRNAs (miRBase 19). To eliminate ambiguous multiple hits during the mapping procedure, no mismatch was allowed. Previous studies reported that, when mapped back to pre-miRNAs, sequence reads usually carried mismatches preferentially located at their terminal $3^{\prime}$ ends (17-20). This mismatch was named the 3 ' end modification. To determine whether the $3^{\prime}$ end modification patterns differed among libraries, as described in our previous studies (21), we trimmed and collected the terminal 3 ' end mismatches one by one. In addition, the remaining perfect match reads had to be at least $18 \mathrm{nt}$ in length. As a result, we kept reads with no less than 18-nt perfect alignment and 3' end modification patterns.

Classifying non-miRNA reads into different data sets. The sequence reads that may not be mapped back to pre-miRNAs were classified into classes by mapping to acquire different data sets with Bowtie (22) and allowing a single nucleotide variation. The sequences of mRNAs and other ncRNAs were derived from the NCBI RefSeq 47 (23). The tRNA sequences were downloaded from the Genomic tRNA database (24) and the rRNA sequences were downloaded from the SILVA database (25). The snoRNA, scaRNA and snRNA sequences were all downloaded from NONCODE (26). The sequence reads not belonging to any of the described RNA classes were uploaded to the RepeatMasker to identify repeat elements, which were classified as unknown.

miRNA expression level according to The Cancer Genome Atlas (TCGA) data. TCGA project collects both cancer and corresponding normal tissues from hundreds of prostate cancer patients. We downloaded all level-3 miRNA expression data of prostate adenocarcinoma from the TCGA Data Portal (https://tcga-data.nci.nih.gov/tcga/dataAccessMatrix. htm). These level-3 data included calculated expressions for each miRNA derived from the Illumina HiSeq sequencing results. A total of 198 tumor samples and 50 normal samples were found at the time the data were downloaded. We kept only the expression data of 50 participants who had both miRNA expression levels from both tumor and normal tissues. Normalized quantification expression levels for these 50 participants were further examined for each investigated miRNA.

Pathway enrichment analysis. We attempted to determine the functions of the miRNA target genes by investigating the pathways with which the miRNA target genes were involved. Therefore, we first downloaded the target genes of differentially expressed miRNAs from TargetScan 6.0, and then mapped the target genes onto the Kyoto Encyclopedia of Genes and Genomes (KEGG) pathways based on the Enzyme Commission (EC) numbers by using the R package SubPathwayMiner v.3.1 (27). Subsequently, the hypergeometric test was performed to identify significantly enriched pathways and calculate the false positive discovery rate in the FDR-corrected q-value.

\section{Results}

miRNA profiling of radiation-treated prostate cancer cells. To characterize the mechanism involved in the radiation 

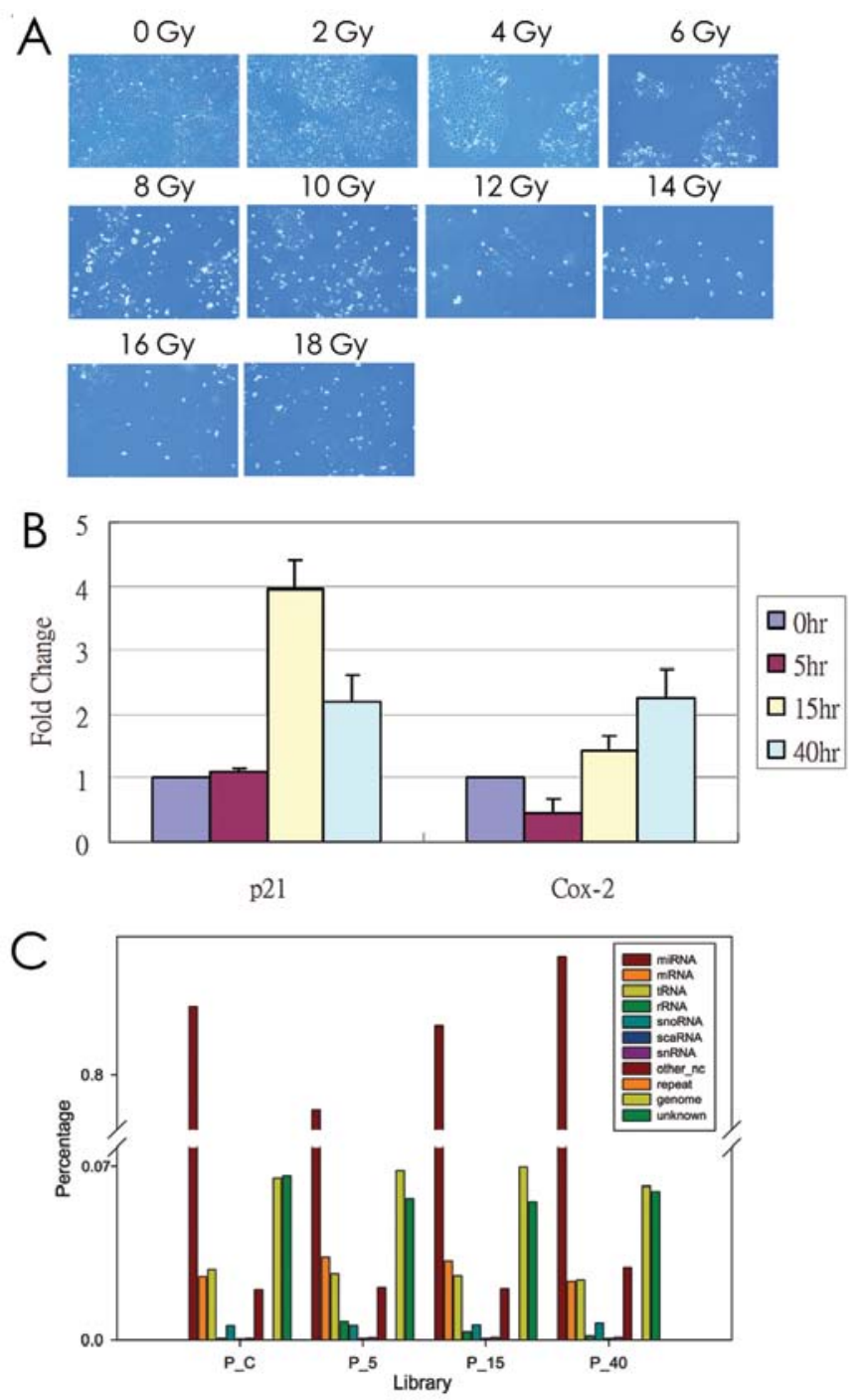

Figure 1. Radiation treatment of human prostate cancer cells, PC3. (A) PC3 cells were treated with various radiation doses $(0,2,4,6,8,10,12,14$, 16 and $18 \mathrm{~Gy}$ ) and were subsequently subjected to fresh culture medium. After culturing for an additional 4 days, the morphology was observed using light microscopy (x40 magnification). (B) The expression pattern of COX-2 and $\mathrm{p} 21$ in radiation-treated PC3 cells was examined using a real-time PCR method. S26 was used as an internal control. (C) The distribution of small RNA reads in 11 categories was classified.

response of prostate cancer, we used NGS to comprehensively analyze the distribution of miRNAs after radiation treatment in PC3 cells. As indicated in Fig. 1A, PC3 cells were exposed to various dosages of radiation $(0,2,4,6,8,10$, $12,14,16$ and $18 \mathrm{~Gy}$ ) and then subjected to a fresh culture medium for an additional 4 days. We found that the growth of the PC3 cells obviously decreased when exposed to $10 \mathrm{~Gy}$ of radiation. Therefore, we collected cell RNA at various times $(0,5,15$ and $40 \mathrm{~h})$ following the $10-\mathrm{Gy}$ radiation treatment. We confirmed the expression levels of Cox-2 and p21, which may be induced by radiation at $24 \mathrm{~h}$ according to previous studies $(16,28)$. The expression levels of Cox-2 and p21 may be upregulated by radiation treatment in PC3 cells (Fig. 1B). We then performed the comprehensive miRNA profile at various time-points in radiation-treated PC3 cells by using the Illumina HiSeq platform.
Table I. Summary of sequence reads and the detected miRNAs.

\begin{tabular}{lcccc}
\hline Library & $\begin{array}{c}\text { Clean } \\
\text { read }(\mathrm{n})\end{array}$ & $\begin{array}{c}\text { miRNA } \\
\text { read }(\%)\end{array}$ & $\begin{array}{c}\text { pre-miRNA } \\
(\mathrm{n})\end{array}$ & $\begin{array}{c}\text { miRNA } \\
(\mathrm{n})\end{array}$ \\
\hline P_C & $9,482,400$ & 80.49 & 693 & 916 \\
P_5 & $9,748,570$ & 79.75 & 687 & 915 \\
P_15 & $9,589,440$ & 80.35 & 712 & 933 \\
P_40 & $10,589,934$ & 80.86 & 739 & 964 \\
\hline
\end{tabular}

P_C, P_5, P_15 and P_40 are prostate cancer cell lines with different radiation treatment. By mapping the clean sequence reads back to pre-miRNAs, we can quantify how many pre-miRNAs and mature miRNAs were detected.

Table II. miRNAs with altered expression in response to radiation in PC3 cells using next-generation sequencing.

\section{$0 \mathrm{~h} \quad 5 \mathrm{~h} \quad 15 \mathrm{~h} \quad 40 \mathrm{~h} \quad$ for TCGA}

\begin{tabular}{llllll}
\hline Upregulation $^{\mathrm{a}}$ & & & & & \\
hsa-miR-9-5p & 1 & 2.82 & 0.90 & 2.63 & \\
hsa-miR-22-3p & 1 & 1.60 & 2.85 & 2.73 & \\
hsa-miR-25-3p & 1 & 1.54 & 1.39 & 2.33 & Upregulation $^{\mathrm{d}}$ \\
hsa-miR-30a-5p & 1 & 2.81 & 2.43 & 2.33 & Upregulation $^{\mathrm{c}}$ \\
hsa-miR-550a-3p & 1 & 1.88 & 1.46 & 2.09 & Upregulation $^{\mathrm{d}}$ \\
hsa-miR-548h-5p & 1 & 0.50 & 0.44 & 2.56 & \\
Downregulation & & & & & \\
hsa-let-7c & 1 & 0.93 & 0.78 & 0.45 & \\
hsa-let-7d-5p & 1 & 0.30 & 0.11 & 0.40 & Upregulation $^{\mathrm{d}}$ \\
hsa-let-7e-5p & 1 & 0.58 & 0.55 & 0.40 & \\
hsa-miR-15a-5p & 1 & 0.78 & 0.67 & 0.45 & Upregulation $^{\mathrm{d}}$ \\
hsa-miR-17-3p & 1 & 0.52 & 0.49 & 0.47 & Upregulation $^{\mathrm{d}}$ \\
hsa-miR-30d-3p & 1 & 0.92 & 0.75 & 0.41 & Upregulation $^{\mathrm{d}}$ \\
hsa-miR-92a-5p & 1 & 0.65 & 0.52 & 0.50 & Upregulation $^{\mathrm{d}}$ \\
hsa-miR-125a-3p & 1 & 0.42 & 0.42 & 0.32 & \\
hsa-miR-197-3p & 1 & 0.77 & 0.79 & 0.44 & Upregulation $^{\mathrm{d}}$ \\
hsa-miR-221-5p & 1 & 0.59 & 0.31 & 0.44 & Downregulation $^{\mathrm{d}}$ \\
hsa-miR-320b & 1 & 0.65 & 0.41 & 0.32 & Upregulation $^{\mathrm{d}}$ \\
hsa-miR-342-5p & 1 & 0.59 & 0.63 & 0.47 & Upregulation $^{\mathrm{d}}$ \\
hsa-miR-361-3p & 1 & 0.45 & 0.53 & 0.40 & Upregulation $^{\mathrm{d}}$ \\
hsa-miR-374a-5p & 1 & 1.04 & 0.93 & 0.47 & \\
hsa-miR-501-3p & 1 & 0.77 & 0.48 & 0.45 & Upregulation $^{\mathrm{d}}$ \\
hsa-miR-671-3p & 1 & 0.77 & 0.62 & 0.41 & Upregulation $^{\mathrm{d}}$ \\
\hline
\end{tabular}

${ }^{a}$ Expression levels of miRNA were inducted $>2$-fold change after PC3 radiation treatment with $10 \mathrm{~Gy}$ for $40 \mathrm{~h}$. ${ }^{\mathrm{b}}$ Expression levels of miRNA were repressed $>2$-fold change after PC 3 radiation treatment with $10 \mathrm{~Gy}$ for $40 \mathrm{~h} .^{\mathrm{c}, \mathrm{d}}$ The difference was indicated to be significant with p-value less than 0.01 or 0.001 .

Analysis of miRNA sequence reads. Once the samples were sequenced, we collected $>9$ million clean reads in all libraries (Table I). In addition to miRNA, we also determined 

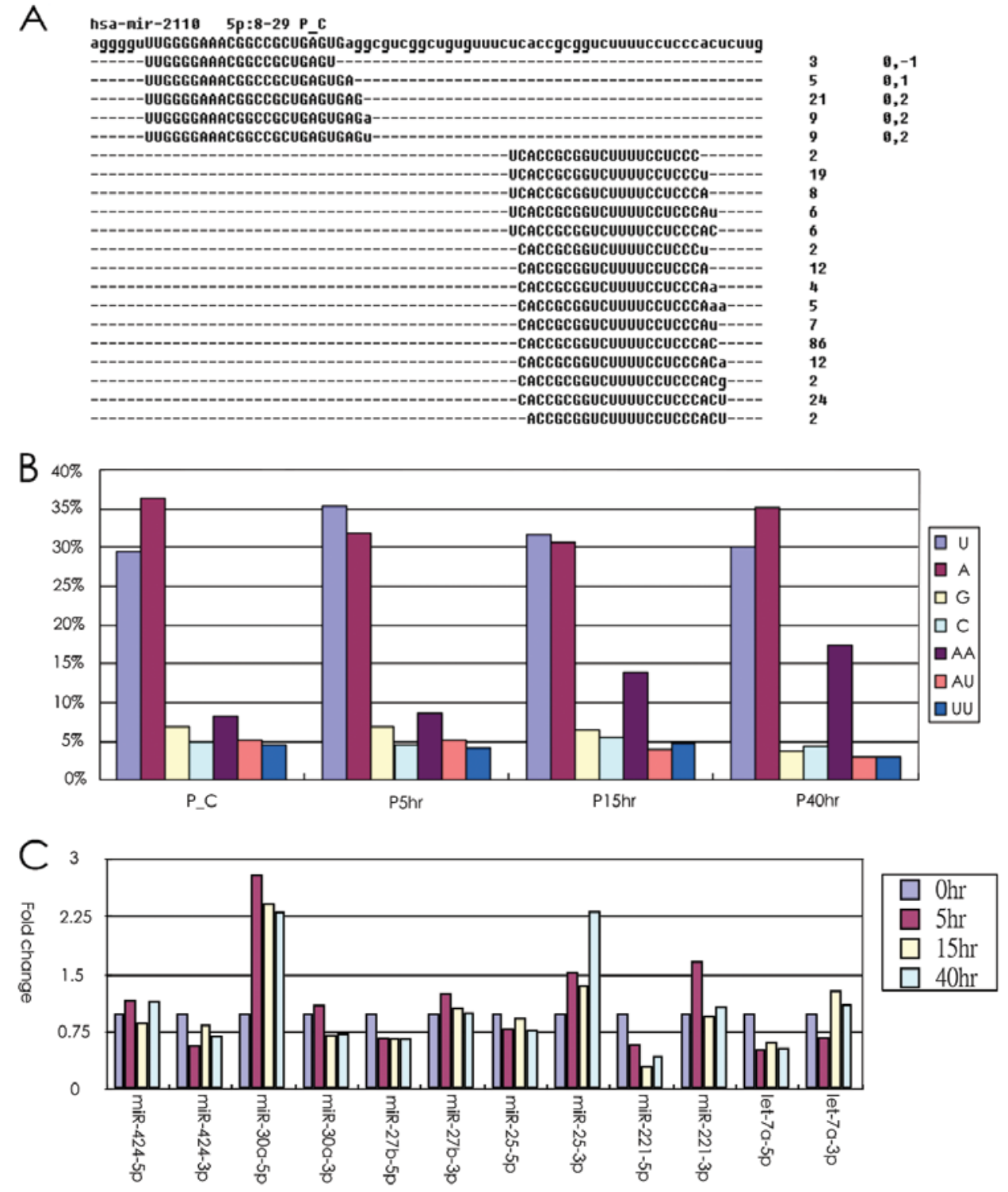

Figure 2. The distribution of isomiRs and -5p/-3p arm selection in PC3 radiation treatment. (A) Mapping results of hsa-miR-2110. As shown in 5p:8-29, hsamiR-2110 encodes mature miRNA at only its $5 \mathrm{p}$ arm, and miRNA spans from nucleotide 8 to nucleotide 29 of the hairpin. The integer values on the left denote the read count of each isomiR. The comma-separated values denote the position shift in the isomiR relative to the miRBase annotated positions ( 8 to 29$)$. The nucleotides in lowercase type denote the sequence fragments originating from the 3 ' modification event. (B) The proportion of 3 ' modifications at end of reads at different time-points after PC 3 radiation treatment as observed from NGS data. (C) Fold-change of the -5p/-3p arm of miRNA at different time points after PC3 radiation treatment as observed from NGS data. isomiRs, miRNA isoforms; miRNA, microRNA; NGS, next-generation sequencing.

which molecules were the remaining non-miRNA reads. By mapping the non-miRNA reads back to a different data set, we classified the reads into 11 categories. Fig. $1 \mathrm{C}$ demonstrates that miRNA accounted for $80 \%$ of all clean reads in the prostate cell libraries. Other categories accounted for relatively low proportions, which indicated the high performance of the sample preparation protocol. In addition, the proportions of the categories were considerably similar among libraries, indicating that radiation treatment did not alter the composition of RNA samples in the prostate cell libraries.

After mapping the clean reads to the genome, most of the miRNA reads tend to exist as isomiR. As demonstrated in Fig. 2A, hsa-miR-2110-5p had 5 isomers, whereas the opposite-arm miRNA-3p had 15 isomiRs, which demonstrated that abundant miRNAs tend to have more isomiRs. Our data revealed that the isomiR quantity was highly correlated with
miRNA abundance (Pearson's correlation coefficient, 0.91). In addition, we observed that the modified nucleotides were preferentially located at the $3^{\prime}$ end of the sequence read (presented in lower case in Fig. 2A). The data indicated that one A nucleotide or one $U$ nucleotide was frequently added at the end of the read. Notably, we found that the proportion of AA dinucleotides modified at the end of the read was gradually increased in a time-dependent manner after the PC3 cells were treated with radiation, which indicated that the 3 ' end modification may be altered by radiation treatment in PC3 cells. Our previous studies indicated that the use of miR-5p and $-3 p$ may be altered in human cancer (29-31). In the present study, our data indicated that arm selection preference was consistent across nearly all libraries. Only a few cases were observed in which the use of $-5 p$ and $-3 p$ arm selection had different preferences at various time-points after radiation 

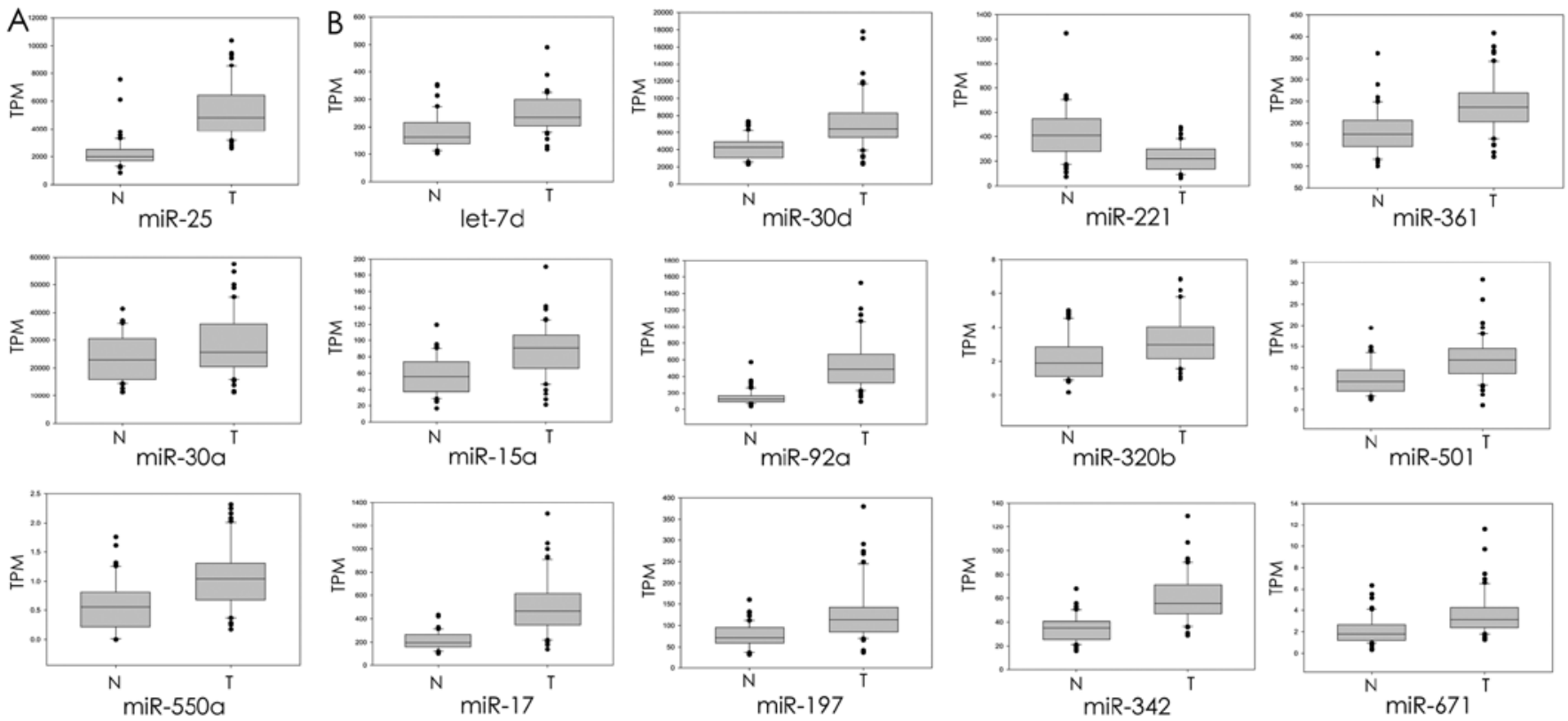

Figure 3. (A) Expression levels of radiation-induced miRNAs in prostate cancer. (B) Expression levels of radiation-suppressed miRNAs in prostate cancer. Expression levels of miRNAs between tumor and corresponding normal tissues from 50 prostate cancer patients were analyzed using TCGA dataset. The expression levels of miRNAs were presented in transcript per million (TPM). The expression level between tumor and normal cells was evaluated by conducting paired t-tests $\left(\mathrm{P}<0.05\right.$ was considered significant; NS, non-significant. $\left.{ }^{*} \mathrm{P}<0.05,{ }^{* *} \mathrm{P}<0.01,{ }^{* * *} \mathrm{P}<0.001\right)$. TCGA, The Cancer Genome Atlas.

treatment (Fig. 2C). Further research is required to support these findings.

Radiation-response miRNAs in prostate cancer. By summarizing the read count of all the isomiRs that belonged to the same mature miRNAs, we quantified the miRNA expression abundances, and presented the result in transcript per million (TPM). Twenty-two miRNAs were selected and are presented in Table II, demonstrating that their expression levels were altered $>2$-fold after being subjected to radiation exposure (expression of 6 miRNAs increased, and expression of 16 miRNAs decreased). To explore the putative role contributing to prostate cancer progression, we examined the effect of expression levels of radiation-associated miRNAs on prostate cancer from the available TCGA dataset by using an in silico analysis. We downloaded 100 miRNA expression profiles from 50 prostate cancer patients, including 50 cancer lesion and 50 corresponding normal tissues. As demonstrated in Fig. 3A, the expression levels of radiation-induced miRNAs, miR-25, miR-30a and miR-550a, were significantly upregulated in the prostate cancer cells compared with the corresponding normal tissue cells. Twelve radiation-suppressed miRNAs were identified, i.e. let-7d, miR-15a, miR-17, miR-30d, miR-92a, miR-197, miR-221, miR-320b, miR-342, miR-361, miR-501 and miR-671, and a significantly different expression between prostate cancer and the corresponding adjacent part was found, including 11 upregulated and 1 downregulated (Fig. 3B). Overall, the data indicated that most of the radiation-response miRNAs were identified as dysregulated in prostate cancer according to an in silico analysis (15/22; 1 downregulated, 14 upregulated and the rest demonstrated no change in expression in prostate cancer).

Pathway enrichment analysis of miRNAs. miRNAs can function as either oncogenes or tumor suppressors depending on their target genes. Therefore, identifying a target can facilitate elucidating the role of miRNAs in prostate cancer treatment radiation $(32,33)$. Typically, one miRNA tends to have hundreds of target genes and a group of miRNAs co-modulated as a biological function involved in the regulation of a signaling pathway. Therefore, we further explored the biological function of radiation-response miRNAs by conducting a pathwayenrichment analysis. The putative target genes of miRNAs were obtained from TargetScan 6.0; subsequently, these target genes of the individual miRNAs were mapped onto KEGG pathways. Our data indicated that the target genes of radiationresponse miRNAs were frequently significantly enriched in several cancer- or radiation-related pathways, including the mitogen-activated protein kinase (MAPK), ErbB, p53, Wnt, transforming growth factor- $\beta$ (TGF- $\beta$ ) and mTOR signaling pathways with an $F D R<0.05$ (Table III). We also subjected the target genes of the 2-gene set, upregulated miRNA and downregulated miRNAs, to pathway-enrichment analysis. Similar results were observed; their targets were significantly enriched in the prostate cancer pathway (Fig. 4) and radiationrelated pathways, including the MAPK, ErbB, Wnt and TGF- $\beta$ signaling pathways (Tables IV and V).

\section{Discussion}

Our previous studies indicated that the distributions of $3^{\prime}$ end modifications and the arm selection preference of miRNAs were different between normal and tumor tissues (29-31). The $-5 p$ and $-3 p$ of miRNA play a distant role by suppressing the different target genes. It was previously reported that, in contrast to the oncogenic effect of miR-17 (-5p), miR-17*(-3P) plays a tumor suppressive role in prostate cancer $(9,34,35)$. The miR-28-5p and miR-28-3p also play opposite roles in colon cancer cell proliferation and migration (36). In the present study, our data showed that the $-5 p$ and $-3 p$ of particular 
Table III. The enriched pathways of radiation-induced miRNA target genes.

\begin{tabular}{|c|c|}
\hline microRNA & Cancer-relative pathway $(\mathrm{FDR}<0.05)$ \\
\hline $\begin{array}{l}\text { Upregulation } \\
\text { hsa-miR-9-5p }\end{array}$ & $\begin{array}{l}\text { Focal adhesion, pathways in cancer, ErbB signaling pathway, MAPK signaling pathway, } \\
\text { prostate cancer }\end{array}$ \\
\hline hsa-miR-22-3p & $\begin{array}{l}\text { Chronic myeloid leukemia, MAPK signaling pathway, ErbB signaling pathway, pathways } \\
\text { in cancer, glioma, prostate cancer, phosphatidylinositol signaling system, colorectal cancer }\end{array}$ \\
\hline hsa-miR-25-3p & N.D \\
\hline hsa-miR-30a-5p & N.D \\
\hline hsa-miR-550a-3p & N.D \\
\hline hsa-miR-548h-5p & N.D \\
\hline \multicolumn{2}{|l|}{ Downregulation } \\
\hline hsa-let-7c & $\begin{array}{l}\text { MAPK signaling pathway, pathways in cancer, } \mathbf{p 5 3} \text { signaling pathway, melanoma, } \\
\text { chronic myeloid leukemia, glioma, pancreatic cancer, focal adhesion, small cell lung cancer, } \\
\text { bladder cancer, prostate cancer }\end{array}$ \\
\hline hsa-let-7d-5p & $\begin{array}{l}\text { MAPK signaling pathway, pathways in cancer, } \mathbf{p 5 3} \text { signaling pathway, melanoma, } \\
\text { chronic myeloid leukemia, glioma, pancreatic cancer, focal adhesion, small cell lung cancer, } \\
\text { bladder cancer, prostate cancer }\end{array}$ \\
\hline hsa-let-7e-5p & $\begin{array}{l}\text { MAPK signaling pathway, pathways in cancer, p53 signaling pathway, melanoma, } \\
\text { chronic myeloid leukemia, glioma, pancreatic cancer, focal adhesion, small cell lung cancer, } \\
\text { bladder cancer, prostate cancer }\end{array}$ \\
\hline hsa-miR-15a-5p & $\begin{array}{l}\text { Pathways in cancer, regulation of actin cytoskeleton, renal cell carcinoma, MAPK signaling pathway, } \\
\text { focal adhesion, melanoma, prostate cancer, Wnt signaling pathway, p53 signaling pathway, } \\
\text { mTOR signaling pathway, non-small cell lung cancer, pancreatic cancer, cell cycle }\end{array}$ \\
\hline hsa-miR-17-3p & $\begin{array}{l}\text { MAPK signaling pathwav, pathways in cancer, chronic myeloid leukemia, pancreatic cancer, } \\
\text { melanoma, bladder cancer, TGF- } \beta \text { signaling pathway, prostate cancer, mTOR signaling pathway, } \\
\text { non-small cell lung cancer, renal cell carcinoma, cell cycle, } \mathbf{p 5 3} \text { signaling pathway }\end{array}$ \\
\hline hsa-miR-30d-3p & N.D \\
\hline hsa-miR-92a-5p & N.D \\
\hline hsa-miR-125a-3p & MAPK signaling pathway, adherens junction, pancreatic cancer, TGF- $\beta$ signaling pathway \\
\hline hsa-miR-197-3p & N.D \\
\hline hsa-miR-221-5p & Wnt signaling pathway, ErbB signaling pathway \\
\hline hsa-miR-320b & $\begin{array}{l}\text { Chronic myeloid leukemia, non-small cell lung cancer, glioma, pathways in cancer, focal adhesion, } \\
\text { pancreatic cancer, melanoma, ErbB signaling pathway, colorectal cancer, TGF- } \beta \text { signaling pathway, } \\
\text { prostate cancer, MAPK signaling pathway, mTOR signaling pathway }\end{array}$ \\
\hline hsa-miR-342-5p & N.D \\
\hline hsa-miR-361-3p & $\begin{array}{l}\text { Pathways in cancer, } \underline{\text { mTOR signaling pathway, }} \text {, melanogenesis, renal cell carcinoma, } \\
\text { nucleotide excision repair }\end{array}$ \\
\hline hsa-miR-374a-5p & $\begin{array}{l}\text { Pathways in cancer, prostate cancer, TGF- } \beta \text { signaling pathway, endometrial cancer, } \\
\text { non-small cell lung cancer, basal cell carcinoma, MAPK signaling pathway }\end{array}$ \\
\hline hsa-miR-501-3p & N.D \\
\hline hsa-miR-671-3p & N.D \\
\hline
\end{tabular}

miRNAs were differently regulated by radiation (shown in Fig. 2C). Several studies have demonstrated that miRNAs contain various ends, which were caused by either RNA editing or non-template nucleotide additions $(17,18,20)$. These miRNA isoforms (isomiRs) contribute to increased miRNA stability or strengthened miRNA-target gene interaction and are differentially expressed in different cellular conditions, including cancer $(16,37,38)$. Our data revealed that the proportion of AA dinucleotide modifications at the end of the read gradually increased in a time-dependent manner after the PC3 cells were treated with radiation, suggesting that radiation may influence the particular miRNA stability or efficiency of silencing targets by regulating the 3 ' end modifications, which warrants further research.

miRNAs are known to function as gene silencers and are involved in modulating biological functions, including cell growth, apoptosis, the cell cycle and the metastasis of cancer (39). Comprehensive miRNA profiling of prostate cancer has indicated that several miRNAs are differentially expressed between prostate cancer and the adjacent normal, which contributes to prostate cancer progression (40-42). In the present study, we analyzed miRNA expression from 


\section{A masare cantar}

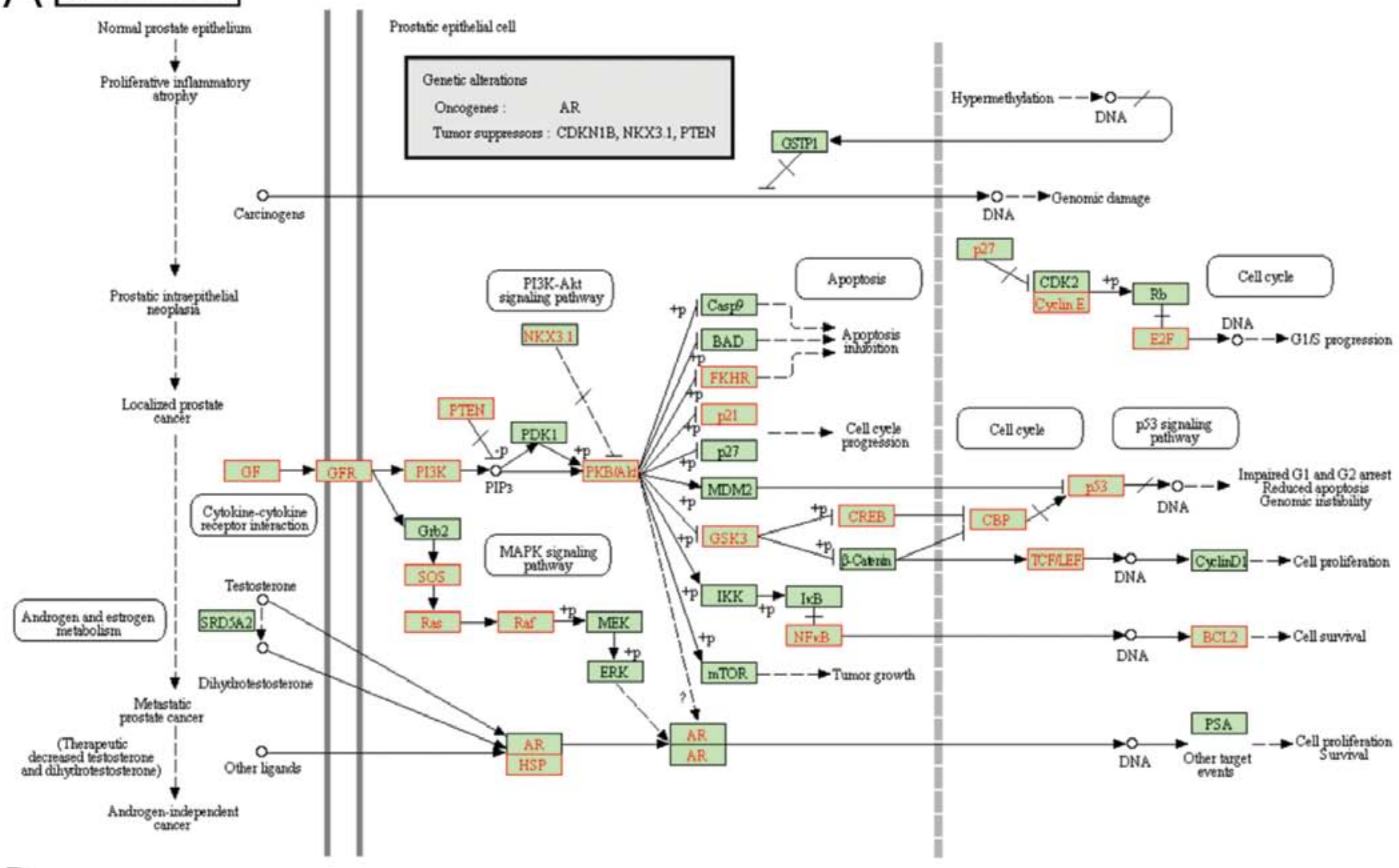

\section{B PROSTATE CANCER}

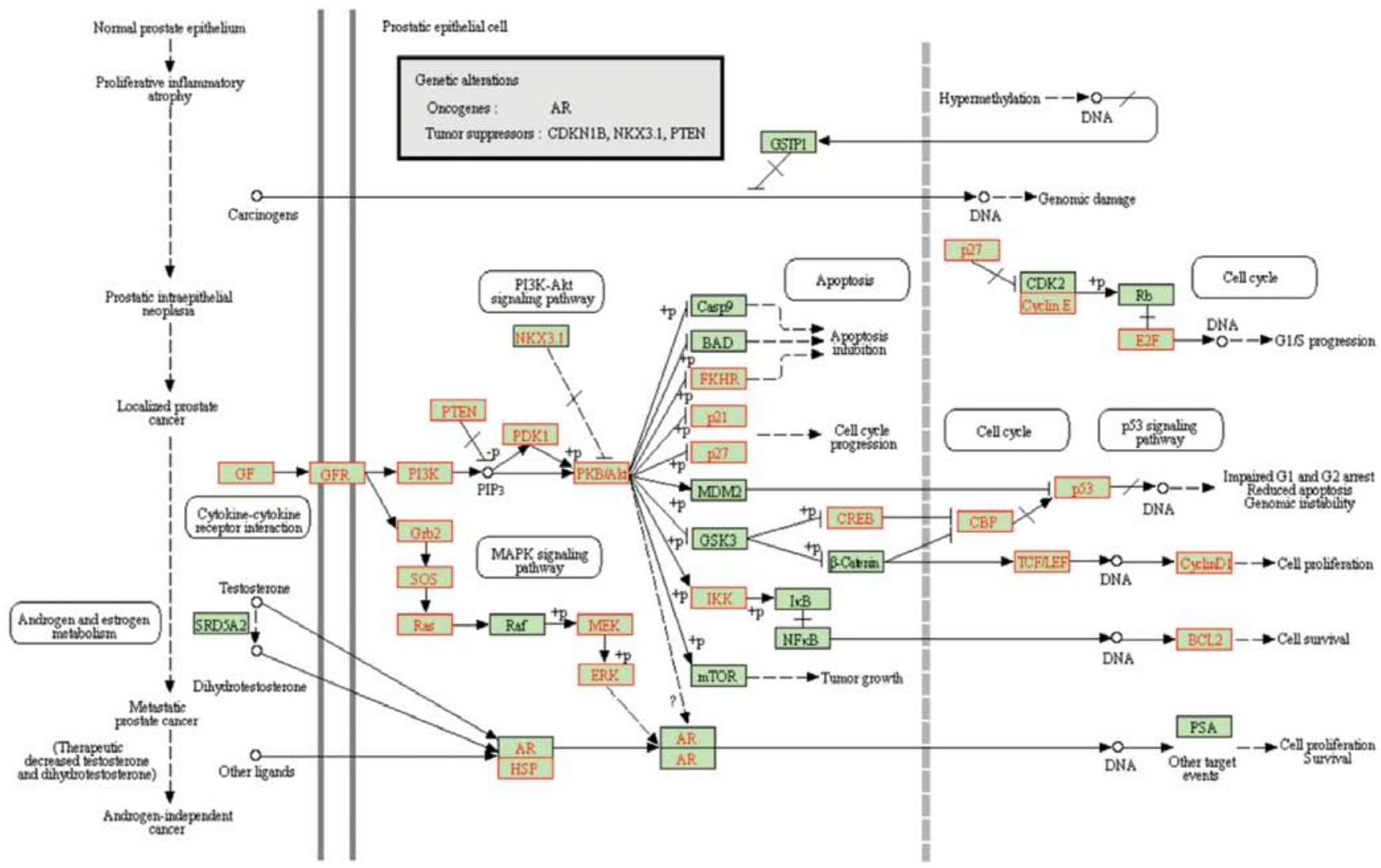

Figure 4. The enriched pathway of the target gene union of radiation-response miRNAs. (A) The target gene union of radiation-upregulated miRNAs enriched in the prostate cancer pathway $(F D R=0.001)$. (B) The target gene union of radiation-downregulated miRNAs enriched in the prostate cancer pathway $(F D R=3.6 \mathrm{E}-9)$. The target genes are labeled in red. 
Table IV. The enriched pathways of radiation-upregulated miRNA target genes.

\begin{tabular}{|c|c|c|}
\hline Pathway Id & Pathway name & FDR \\
\hline Path:04360 & Axon guidance & 7.88E-08 \\
\hline Path:04722 & Neurotrophin signaling pathway & $1.11 \mathrm{E}-07$ \\
\hline Path:04010 & MAPK signaling pathway & $1.11 \mathrm{E}-07$ \\
\hline Path:05200 & Pathways in cancer & $3.25 \mathrm{E}-07$ \\
\hline Path:04012 & ErbB signaling pathway & $3.14 \mathrm{E}-06$ \\
\hline Path:04120 & Ubiquitin mediated proteolysis & $8.82 \mathrm{E}-06$ \\
\hline Path:04144 & Endocytosis & $1.24 \mathrm{E}-05$ \\
\hline Path:04520 & Adherens junction & $5.86 \mathrm{E}-05$ \\
\hline Path:05412 & Arrhythmogenic right ventricular cardiomyopathy (ARVC) & 7.26E-05 \\
\hline Path:04510 & Focal adhesion & 0.000105 \\
\hline Path:04916 & Melanogenesis & 0.000245 \\
\hline Path:05220 & Chronic myeloid leukemia & 0.000245 \\
\hline Path:04810 & Regulation of actin cytoskeleton & 0.000261 \\
\hline Path:05214 & Glioma & 0.000261 \\
\hline Path:05414 & Dilated cardiomyopathy & 0.000268 \\
\hline Path:04910 & Insulin signaling pathway & 0.000282 \\
\hline Path:04720 & Long-term potentiation & 0.000294 \\
\hline Path:04070 & Phosphatidylinositol signaling system & 0.000294 \\
\hline Path:05410 & Hypertrophic cardiomyopathy (HCM) & 0.000802 \\
\hline Path:05100 & Bacterial invasion of epithelial cells & 0.001018 \\
\hline Path:05215 & $\underline{\text { Prostate cancer }}$ & 0.001096 \\
\hline Path:04310 & Wnt signaling pathway & 0.001153 \\
\hline Path:04914 & Progesterone-mediated oocyte maturation & 0.002548 \\
\hline Path:05211 & Renal cell carcinoma & 0.003135 \\
\hline Path:04920 & Adipocytokine signaling pathway & 0.003902 \\
\hline Path:04350 & TGF- $\beta$ signaling pathway & 0.003902 \\
\hline Path:04666 & Fc $\gamma$ R-mediated phagocytosis & 0.004314 \\
\hline Path:04141 & Protein processing in endoplasmic reticulum & 0.005738 \\
\hline Path:05210 & Colorectal cancer & 0.005747 \\
\hline Path:04130 & SNARE interactions in vesicular transport & 0.007624 \\
\hline Path:05216 & Thyroid cancer & 0.008679 \\
\hline Path:00562 & Inositol phosphate metabolism & 0.008679 \\
\hline Path:00532 & Glycosaminoglycan biosynthesis-chondroitin sulfate & 0.008679 \\
\hline Path:05014 & Amyotrophic lateral sclerosis (ALS) & 0.008679 \\
\hline Path:05212 & Pancreatic cancer & 0.008679 \\
\hline Path:04020 & Calcium signaling pathway & 0.008679 \\
\hline Path:05218 & Melanoma & 0.010147 \\
\hline Path:04930 & Type II diabetes mellitus & 0.018227 \\
\hline Path:04962 & Vasopressin-regulated water reabsorption & 0.018227 \\
\hline Path:04530 & Tight junction & 0.019289 \\
\hline Path:04512 & ECM-receptor interaction & 0.019289 \\
\hline Path:04912 & GnRH signaling pathway & 0.019289 \\
\hline Path:05223 & Non-small cell lung cancer & 0.023349 \\
\hline Path:05222 & Small cell lung cancer & 0.032425 \\
\hline Path:05213 & Endometrial cancer & 0.035464 \\
\hline Path:04662 & B cell receptor signaling pathway & 0.035464 \\
\hline Path:05131 & Shigellosis & 0.035464 \\
\hline Path:05221 & Acute myeloid leukemia & 0.03804 \\
\hline Path:04540 & Gap junction & 0.041068 \\
\hline Path:00250 & Alanine, aspartate and glutamate metabolism & 0.045237 \\
\hline Path:04114 & Oocyte meiosis & 0.049286 \\
\hline
\end{tabular}


Table V. The enriched pathways of radiation-downregulated miRNA target genes.

\begin{tabular}{|c|c|c|}
\hline Pathway Id & Pathway name & FDR \\
\hline Path:04010 & MAPK signaling pathway & 0 \\
\hline Path:04360 & Axon guidance & 0 \\
\hline Path:05200 & Pathways in cancer & 0 \\
\hline Path:04722 & Neurotrophin signaling pathway & $1.18 \mathrm{E}-12$ \\
\hline Path:04310 & Wnt signaling pathway & $1.50 \mathrm{E}-10$ \\
\hline Path:04510 & Focal adhesion & $4.27 \mathrm{E}-10$ \\
\hline Path:04144 & Endocytosis & $1.40 \mathrm{E}-09$ \\
\hline Path:04810 & Regulation of actin cytoskeleton & 2.34E-09 \\
\hline Path:05215 & $\underline{\text { Prostate cancer }}$ & $3.58 \mathrm{E}-09$ \\
\hline Path:05211 & Renal cell carcinoma & $3.58 \mathrm{E}-09$ \\
\hline Path:04720 & Long-term potentiation & $1.14 \mathrm{E}-08$ \\
\hline Path:05220 & Chronic myeloid leukemia & $3.41 \mathrm{E}-08$ \\
\hline Path:04120 & Ubiquitin mediated proteolysis & 7.40E-08 \\
\hline Path:04020 & Calcium signaling pathway & $1.10 \mathrm{E}-07$ \\
\hline Path:05212 & Pancreatic cancer & $1.57 \mathrm{E}-07$ \\
\hline Path:05214 & Glioma & $1.59 \mathrm{E}-07$ \\
\hline Path:05218 & Melanoma & $2.36 \mathrm{E}-07$ \\
\hline Path:05223 & Non-small cell lung cancer & $3.64 \mathrm{E}-07$ \\
\hline Path:04916 & Melanogenesis & 4.67E-07 \\
\hline Path:04520 & Adherens junction & 4.67E-07 \\
\hline Path:04910 & Insulin signaling pathway & $6.79 \mathrm{E}-07$ \\
\hline Path:04350 & TGF- $\beta$ signaling pathway & 7.72E-07 \\
\hline Path:05210 & Colorectal cancer & $2.50 \mathrm{E}-06$ \\
\hline Path:04012 & ErbB signaling pathway & 3.89E-06 \\
\hline Path:05222 & Small cell lung cancer & $3.20 \mathrm{E}-05$ \\
\hline Path:04730 & Long-term depression & $5.13 \mathrm{E}-05$ \\
\hline Path:05221 & Acute myeloid leukemia & 7.50E-05 \\
\hline Path:04150 & mTOR signaling pathway & $8.68 \mathrm{E}-05$ \\
\hline Path:05213 & Endometrial cancer & 8.68E-05 \\
\hline Path:05217 & Basal cell carcinoma & $9.44 \mathrm{E}-05$ \\
\hline Path:04710 & Circadian rhythm-mammal & $9.64 \mathrm{E}-05$ \\
\hline Path:04070 & Phosphatidylinositol signaling system & $9.64 \mathrm{E}-05$ \\
\hline Path:04540 & Gap junction & 9.83E-05 \\
\hline Path:04115 & p53 signaling pathway & 0.000113 \\
\hline Path:04141 & Protein processing in endoplasmic reticulum & 0.000137 \\
\hline Path:04114 & Oocyte meiosis & 0.000196 \\
\hline Path:05014 & Amyotrophic lateral sclerosis (ALS) & 0.000353 \\
\hline Path:04970 & Salivary secretion & 0.000414 \\
\hline Path:04660 & $\mathrm{T}$ cell receptor signaling pathway & 0.000449 \\
\hline Path:04666 & Fc $\gamma$ R-mediated phagocytosis & 0.000526 \\
\hline Path:04512 & ECM-receptor interaction & 0.000526 \\
\hline Path:05142 & Chagas disease & 0.000563 \\
\hline Path:04062 & Chemokine signaling pathway & 0.000812 \\
\hline Path:04210 & $\underline{\text { Apoptosis }}$ & 0.000844 \\
\hline Path:04914 & Progesterone-mediated oocyte maturation & 0.001465 \\
\hline Path:04110 & Cell cycle & 0.001527 \\
\hline Path:04662 & B cell receptor signaling pathway & 0.00169 \\
\hline Path:04530 & Tight junction & 0.001692 \\
\hline Path:04930 & Type II diabetes mellitus & 0.002128 \\
\hline Path:05412 & Arrhythmogenic right ventricular cardiomyopathy (ARVC) & 0.002128 \\
\hline Path:04920 & Adipocytokine signaling pathway & 0.002184 \\
\hline Path:04664 & Fc $\varepsilon$ RI signaling pathway & 0.002621 \\
\hline
\end{tabular}


Table V. Continued.

\begin{tabular}{|c|c|c|}
\hline Pathway Id & Pathway name & FDR \\
\hline Path:04960 & Aldosterone-regulated sodium reabsorption & 0.002802 \\
\hline Path:04912 & GnRH signaling pathway & 0.002955 \\
\hline Path:04320 & Dorso-ventral axis formation & 0.00373 \\
\hline Path:05219 & Bladder cancer & 0.00373 \\
\hline Path:04340 & Hedgehog signaling pathway & 0.005218 \\
\hline Path:04971 & Gastric acid secretion & 0.005633 \\
\hline Path:04130 & SNARE interactions in vesicular transport & 0.006926 \\
\hline Path:00532 & Glycosaminoglycan biosynthesis-chondroitin sulfate & 0.006926 \\
\hline Path:05131 & Shigellosis & 0.008185 \\
\hline Path:05160 & Hepatitis C & 0.008185 \\
\hline Path:04630 & Jak-STAT signaling pathway & 0.008368 \\
\hline Path:05410 & Hypertrophic cardiomyopathy (HCM) & 0.009939 \\
\hline Path:05414 & Dilated cardiomyopathy & 0.010733 \\
\hline Path:00534 & Glycosaminoglycan biosynthesis-heparan sulfate & 0.011359 \\
\hline Path:04670 & Leukocyte transendothelial migration & 0.011852 \\
\hline Path:04370 & VEGF signaling pathway & 0.013265 \\
\hline Path:00562 & Inositol phosphate metabolism & 0.013625 \\
\hline Path:04270 & Vascular smooth muscle contraction & 0.014557 \\
\hline Path:00512 & O-Glycan biosynthesis & 0.016472 \\
\hline Path:04330 & Notch signaling pathway & 0.026769 \\
\hline Path:04142 & Lysosome & 0.038882 \\
\hline Path:00533 & Glycosaminoglycan biosynthesis-keratan sulfate & 0.038882 \\
\hline Path:05145 & Toxoplasmosis & 0.048785 \\
\hline
\end{tabular}

TCGA database and found that the expression levels of radiation-induced miRNAs were frequently dysregulated in prostate cancer (Fig. 3). Our results are consistent with those of previous studies and demonstrated that miR-25, miR-17, miR-30d and miR-92a are overexpressed, and miR-221 is downregulated in prostate cancer $(9,42-44)$. However, the expression levels of let-7d and miR-15a decreased according to TCGA, which contradicted the results of previous studies (45-47). These dysfunctional miRNAs have potential to be used as biomarkers for prostate cancer prognosis or diagnosis. Therefore, understanding the function of miRNAs may provide practical benefits for clinical applications. Predicting the outcome of cancer treatment is the most promising application of miRNAs. Gonzales et al found miR-141 to be consistent with changes in other conventional biomarkers and to the clinical outcomes, suggesting that miR-141 can be used as a marker for monitoring therapeutic response in prostate cancer patients (48). The prognostic value of miRNA expression profiling in prostate cancer has also been demonstrated by Hulf et al. They demonstrated that DNA methylation and histone H3K9-deacetylation of the miR-205 locus is associated with miRNA silencing and deregulation of MED1, which is predictive of a poor prognosis in localized prostate cancer (49).

Ionizing radiation is one of the 3 primary modalities used in cancer therapy. Radiation induces considerable DNA damages, which, if not repaired, cause cancer cells to progress to apoptosis and cell cycle arrest. Some cancer cells are resistant to radiation treatment due to activation of complex signaling pathways that counteract these damages, including ErbB, nuclear factor $\kappa \mathrm{B}(\mathrm{NF} \kappa \mathrm{B})$, MAPK, PI3K/AKT and transforming growth factor- $\beta$ (TGF- $\beta$ ) signaling pathways (50-52). Several radiation-related miRNAs have been identified that contribute to the radiosensitivity of cancer cells by modulating the radiation-response signaling pathway $(51,52)$. Since miRNAs are generally slightly repressed by their target genes, the alteration of an individual miRNA is insufficient for accomplishing a biological function. Previous studies have introduced the concept of miRNA regulatory modules (MRMs), which potentially serve as a model for understanding the detailed influences of miRNAs in cellular biological functions (53-55). Therefore, in the present study, we were particularly interested in the consequences of changes in a group of radiation-induced miRNAs in prostate cancer. Our data indicated that targets of the co-expressed miRNAs were enriched in a radiation-related signaling pathway, suggesting that they co-modulated an abundance of target genes in the same pathway (Table III).

miRNAs regulate various factors in radiation-related biological pathways and may affect the radiosensitivity of tumor cells (51). Radiation-response miRNAs have been identified in prostate cancer by using a microarray approach (13-15). By comparing these data, we identified known and unknown radiation-response miRNAs in prostate cancer by using an NGS approach. Li et al reported that the expression levels of miR-9, miR-22 and miR-30a decreased in radiation-treated PC3 cells (14). Radiation reduced the expression level of an miR-17-92a cluster and the let-7 family in prostate cancer (15). 
In the present study, we also identified radiation-response miRNAs that had been reported in other types of cancer but not in prostate cancer, such as miR-25, miR-15a, miR-30d, miR-125a, miR-221 and miR-342 (21,56-63). In addition, we identified a group of radiation-response miRNAs that have not been reported in any type of cancer. The pathway-enrichment analysis revealed that their targets are frequently enriched in the radiation-response signaling pathway.

In summary, in the present study, we thoroughly investigated radiation-response miRNAs, which may be involved in the radiosensitivity of prostate cancer, by modulating radiationrelated signaling pathways using an NGS approach. These miRNA candidates may be effective targets for improving the efficacy of radiation treatment in future prostate cancer therapy. In addition, we observed that $3^{\prime}$ end modifications and the $-5 \mathrm{p} /-3 \mathrm{p}$ arm selection of miRNAs were altered in prostate cancer after radiation treatment. These finding require further research.

\section{Acknowledgements}

This study was supported by grants from Kaohsiung Veterans General Hospital (VGHKS 102-005 and VGHKS 102-074). The authors thank Genomics and Proteomics Core Laboratory, Department of Medical Research, Kaohsiung Chang Gung Memorial Hospital, for the assistance with NGS data analysis.

\section{References}

1. Crawford ED: Epidemiology of prostate cancer. Urology 62 (Suppl 1): 3-12, 2003.

2. Bartel DP: MicroRNAs: genomics, biogenesis, mechanism, and function. Cell 116: 281-297, 2004.

3. Krol J, Loedige I and Filipowicz W: The widespread regulation of microRNA biogenesis, function and decay. Nat Rev Genet 11: 597-610, 2010.

4. Selbach M, Schwanhäusser B, Thierfelder N, Fang Z, Khanin R and Rajewsky N: Widespread changes in protein synthesis induced by microRNAs. Nature 455: 58-63, 2008.

5. Porkka KP, Pfeiffer MJ, Waltering KK, Vessella RL, Tammela TL and Visakorpi T: MicroRNA expression profiling in prostate cancer. Cancer Res 67: 6130-6135, 2007.

6. Sevli S, Uzumcu A, Solak M, Ittmann M and Ozen M: The function of microRNAs, small but potent molecules, in human prostate cancer. Prostate Cancer Prostatic Dis 13: 208-217, 2010 .

7. Li T, Li D, Sha J, Sun P and Huang Y: MicroRNA-21 directly targets MARCKS and promotes apoptosis resistance and invasion in prostate cancer cells. Biochem Biophys Res Commun 383: 280-285, 2009.

8. Ambs S, Prueitt RL, Yi M, et al: Genomic profiling of microRNA and messenger RNA reveals deregulated microRNA expression in prostate cancer. Cancer Res 68: 6162-6170, 2008.

9. Sylvestre Y, De Guire V, Querido E, et al: An E2F/miR-20a autoregulatory feedback loop. J Biol Chem 282: 2135-2143, 2007.

10. Fujita Y, Kojima K, Hamada N, et al: Effects of miR-34a on cell growth and chemoresistance in prostate cancer PC3 cells. Biochem Biophys Res Commun 377: 114-119, 2008.

11. Shi XB, Xue L, Yang J, et al: An androgen-regulated miRNA suppresses Bak1 expression and induces androgen-independent growth of prostate cancer cells. Proc Natl Acad Sci USA 104: 19983-19988, 2007

12. Gandellini P, Folini M, Longoni N, et al: miR-205 exerts tumor-suppressive functions in human prostate through downregulation of protein kinase C $\varepsilon$. Cancer Res 69: 2287-2295, 2009.

13. Josson S, Sung SY, Lao K, Chung LW and Johnstone PA: Radiation modulation of microRNA in prostate cancer cell lines Prostate 68: 1599-1606, 2008.

14. Li B, Shi XB, Nori D, et al: Down-regulation of microRNA $106 \mathrm{~b}$ is involved in p21-mediated cell cycle arrest in response to radiation in prostate cancer cells. Prostate 71: 567-574, 2011.
15. John-Aryankalayil M,Palayoor ST,Makinde AY, et al: Fractionated radiation alters oncomir and tumor suppressor miRNAs in human prostate cancer cells. Radiat Res 178: 105-117, 2012.

16. Cloonan $\mathrm{N}$, Wani $\mathrm{S}, \mathrm{Xu} \mathrm{Q}$, et al: MicroRNAs and their isomiRs function cooperatively to target common biological pathways. Genome Biol 12: R126, 2011.

17. Ebhardt HA, Tsang HH, Dai DC, Liu Y, Bostan B and Fahlman RP: Meta-analysis of small RNA-sequencing errors reveals ubiquitous post-transcriptional RNA modifications. Nucleic Acids Res 37: 2461-2470, 2009.

18. Landgraf $\mathrm{P}$, Rusu M, Sheridan R, et al: A mammalian microRNA expression atlas based on small RNA library sequencing. Cell 129: 1401-1414, 2007.

19. Reid JG, Nagaraja AK, Lynn FC, et al: Mouse let-7 miRNA populations exhibit RNA editing that is constrained in the 5'-seed/cleavage/anchor regions and stabilize predicted mmu-let7a:mRNA duplexes. Genome Res 18: 1571-1581, 2008.

20. Morin RD, O'Connor MD, Griffith M, et al: Application of massively parallel sequencing to microRNA profiling and discovery in human embryonic stem cells. Genome Res 18: 610-621, 2008.

21. Chaudhry MA, Omaruddin RA, Brumbaugh CD, Tariq MA and Pourmand N: Identification of radiation-induced microRNA transcriptome by next-generation massively parallel sequencing. J Radiat Res 54: 808-822, 2013.

22. Langmead B, Trapnell C, Pop M and Salzberg SL: Ultrafast and memory-efficient alignment of short DNA sequences to the human genome. Genome Biol 10: R25, 2009.

23. Pruitt KD, Tatusova T, Klimke W and Maglott DR: NCBI Reference Sequences: current status, policy and new initiatives. Nucleic Acids Res 37: D32-D36, 2009.

24. Chan PP and Lowe TM: GtRNAdb: a database of transfer RNA genes detected in genomic sequence. Nucleic Acids Res 37: D93-D97, 2009.

25. Pruesse E, Quast C, Knittel K, et al: SILVA: a comprehensive online resource for quality checked and aligned ribosomal RNA sequence data compatible with ARB. Nucleic Acids Res 35: 7188-7196, 2007.

26. Liu C, Bai B, Skogerbø G, et al: NONCODE: an integrated knowledge database of non-coding RNAs. Nucleic Acids Res 33: D112-D115, 2005.

27. Li C, Li X, Miao Y, et al: SubpathwayMiner: a software package for flexible identification of pathways. Nucleic Acids Res 37: e131, 2009.

28. John-Aryankalayil M, Palayoor ST, Cerna D, et al: Fractionated radiation therapy can induce a molecular profile for therapeutic targeting. Radiat Res 174: 446-458, 2010.

29. Chang HT, Li SC, Ho MR, et al: Comprehensive analysis of microRNAs in breast cancer. BMC Genomics 13 (Suppl 7): S18, 2012.

30. Li SC, Liao YL, Ho MR, Tsai KW, Lai CH and Lin WC: miRNA arm selection and isomiR distribution in gastric cancer. BMC Genomics 13 (Suppl 1): S13, 2012.

31. Li SC, Tsai KW, Pan HW, Jeng YM, Ho MR and Li WH: MicroRNA 3' end nucleotide modification patterns and arm selection preference in liver tissues. BMC Syst Biol 6 (Suppl 2): S14, 2012.

32. Krek A, Grün D, Poy MN, et al: Combinatorial microRNA target predictions. Nat Genet 37: 495-500, 2005.

33. Rehmsmeier M, Steffen P, Hochsmann M and Giegerich R: Fast and effective prediction of microRNA/target duplexes. RNA 10: 1507-1517, 2004.

34. Xu Y, Fang F, Zhang J, Josson S, St Clair WH and St Clair DK: miR-17* suppresses tumorigenicity of prostate cancer by inhibiting mitochondrial antioxidant enzymes. PLoS One 5: e14356, 2010.

35. Zhang X, Ladd A, Dragoescu E, Budd WT, Ware JL and Zehner ZE: MicroRNA-17-3p is a prostate tumor suppressor in vitro and in vivo, and is decreased in high grade prostate tumors analyzed by laser capture microdissection. Clin Exp Metastasis 26: 965-979, 2009.

36. Almeida MI, Nicoloso MS, Zeng L, et al: Strand-specific miR-28-5p and miR-28-3p have distinct effects in colorectal cancer cells. Gastroenterology 142: 886-896, 2012.

37. Fernandez-Valverde SL, Taft RJ and Mattick JS: Dynamic isomiR regulation in Drosophila development. RNA 16: 1881-1888, 2010.

38. Guo L, Li H, Liang T, et al: Consistent isomiR expression patterns and 3 ' addition events in miRNA gene clusters and families implicate functional and evolutionary relationships. Mol Biol Rep 39: 6699-6706, 2012. 
39. Pan HW, Li SC and Tsai KW: MicroRNA dysregulation in gastric cancer. Curr Pharm Des 19: 1273-1284, 2013.

40. Leite KR, Tomiyama A, Reis ST, et al: MicroRNA expression profiles in the progression of prostate cancer - from high-grade prostate intraepithelial neoplasia to metastasis. Urol Oncol 31: 796-801, 2013.

41. Schubert M, Spahn M, Kneitz S, et al: Distinct microRNA expression profile in prostate cancer patients with early clinical failure and the impact of let-7 as prognostic marker in high-risk prostate cancer. PLoS One 8: e65064, 2013.

42. Walter BA, Valera VA, Pinto PA and Merino MJ: Comprehensive microRNA Profiling of Prostate Cancer. J Cancer 4: 350-357, 2013.

43. Kobayashi N, Uemura H, Nagahama $\mathrm{K}$, et al: Identification of miR-30d as a novel prognostic maker of prostate cancer. Oncotarget 3: 1455-1471, 2012.

44. Poliseno L, Salmena L, Riccardi L, et al: Identification of the miR-106b 25 microRNA cluster as a proto-oncogenic PTENtargeting intron that cooperates with its host gene MCM7 in transformation. Sci Signal 3: ra29, 2010.

45. Ramberg H, Alshbib A, Berge V, Svindland A and Taskén KA: Regulation of PBX3 expression by androgen and Let-7d in prostate cancer. Mol Cancer 10: 50, 2011.

46. Bonci D, Coppola V, Musumeci M, et al: The miR-15a-miR-16-1 cluster controls prostate cancer by targeting multiple oncogenic activities. Nat Med 14: 1271-1277, 2008.

47. Porkka KP, Ogg EL, Saramaki OR, et al: The miR-15a-miR-16-1 locus is homozygously deleted in a subset of prostate cancers. Genes Chromosomes Cancer 50: 499-509, 2011.

48. Gonzales JC, Fink LM, Goodman OB Jr, Symanowski JT, Vogelzang NJ and Ward DC: Comparison of circulating microRNA 141 to circulating tumor cells, lactate dehydrogenase, and prostate-specific antigen for determining treatment response in patients with metastatic prostate cancer. Clin Genitourin Cancer 9: 39-45, 2011.

49. Hulf T, Sibbritt T, Wiklund ED, et al: Epigenetic-induced repression of microRNA-205 is associated with MED1 activation and a poorer prognosis in localized prostate cancer. Oncogene 32 : 2891-2899, 2013

50. Runkle EA, Zhang H, Cai Z, et al: Reversion of the ErbB malignant phenotype and the DNA damage response. Exp Mol Pathol 93: 324-333, 2012.
51. Zhao L, Bode AM, Cao Y and Dong Z: Regulatory mechanisms and clinical perspectives of miRNA in tumor radiosensitivity. Carcinogenesis 33: 2220-2227, 2012

52. Zhao L, Lu X and Cao Y: MicroRNA and signal transduction pathways in tumor radiation response. Cell Signal 25: 1625-1634, 2013.

53. Joung JG, Hwang KB, Nam JW, Kim SJ and Zhang BT: Discovery of microRNA-mRNA modules via population-based probabilistic learning. Bioinformatics 23: 1141-1147, 2007.

54. Tran DH, Satou $\mathrm{K}$ and Ho TB: Finding microRNA regulatory modules in human genome using rule induction. BMC Bioinformatics 9 (Suppl 12): S5, 2008.

55. Yoon S and De Micheli G: Prediction of regulatory modules comprising microRNAs and target genes. Bioinformatics 21 (Suppl 2): ii93-ii100, 2005.

56. Chaudhry MA, Sachdeva H and Omaruddin RA: Radiationinduced micro-RNA modulation in glioblastoma cells differing in DNA-repair pathways. DNA Cell Biol 29: 553-561, 2010.

57. Wang Q, Li P, Li A, et al: Plasma specific miRNAs as predictive biomarkers for diagnosis and prognosis of glioma. J Exp Clin Cancer Res 31: 97, 2012.

58. Chaudhry MA and Omaruddin RA: Differential regulation of microRNA expression in irradiated and bystander cells. Mol Biol 46: 634-643, 2012.

59. Vincenti S, Brillante $\mathrm{N}$, Lanza V, et al: HUVEC respond to radiation by inducing the expression of pro-angiogenic microRNAs. Radiat Res 175: 535-546, 2011.

60. Chun-Zhi Z, Lei H, An-Ling Z, et al: MicroRNA-221 and microRNA-222 regulate gastric carcinoma cell proliferation and radioresistance by targeting PTEN. BMC Cancer 10: 367, 2010.

61. Xu Y, Zhou B, Wu D, Yin Z and Luo D: Baicalin modulates microRNA expression in UVB irradiated mouse skin. J Biomed Res 26: 125-134, 2012.

62. Wagner-Ecker M, Schwager C, Wirkner U, Abdollahi A and Huber PE: MicroRNA expression after ionizing radiation in human endothelial cells. Radiat Oncol 5: 25, 2010.

63. Wang Y, Scheiber MN, Neumann C, Calin GA and Zhou D: MicroRNA regulation of ionizing radiation-induced premature senescence. Int J Radiat Oncol Biol Phys 81: 839-848, 2011. 